\title{
Hall drift of axisymmetric magnetic fields in solid neutron-star matter
}

\author{
A. Reisenegger ${ }^{1,2}$, R. Benguria ${ }^{3}$, J. P. Prieto ${ }^{1}$, P. A. Araya ${ }^{1, \star}$, and D. Lai ${ }^{4}$ \\ 1 Departamento de Astronomía y Astrofísica, Pontificia Universidad Católica de Chile, Casilla 306, Santiago 22, Chile \\ e-mail: areisene@astro.puc.cl \\ 2 Max-Planck-Institut für Astrophysik, Karl-Schwarzschild-Str. 1, 85741 Garching bei München, Germany \\ 3 Departamento de Física, Pontificia Universidad Católica de Chile, Casilla 306, Santiago 22, Chile \\ ${ }^{4}$ Center for Radiophysics and Space Research, Department of Astronomy, Cornell University, Ithaca, NY 14853, USA
}

Received 14 May 2007 / Accepted 28 June 2007

\begin{abstract}
Context. The Hall drift, namely, the transport of magnetic flux by the moving electrons giving rise to the electrical current, may be the dominant effect causing the evolution of the magnetic field in the solid crust of neutron stars. It is a nonlinear process that, despite a number of theoretical efforts, is still not fully understood.

Aims. Through mostly analytic arguments and solutions, we intend to help understand this highly nonlinear process.

Methods. We use the Hall induction equation in axial symmetry to obtain some general properties of nonevolving fields, as well as analyzing the evolution of purely toroidal fields, their poloidal perturbations, and current-free, purely poloidal fields. We also analyze energy conservation in Hall instabilities and write down a variational principle for Hall equilibria.

Results. We show that the evolution of any toroidal magnetic field can be described by Burgers' equation, as previously found by Vainshtein and collaborators in a plane-parallel geometry. This evolution leads to sharp current sheets, which dissipate on the Hall time scale, yielding a stationary field configuration that depends on a single, suitably defined coordinate. This field, however, is unstable to poloidal perturbations, which grow as their field lines are stretched by the background electron flow, as in the instabilities found numerically by Rheinhardt and Geppert. On the other hand, current-free poloidal configurations are stable and could represent a long-lived crustal field supported by currents in the fluid stellar core. There may be additional, stable configurations, corresponding to restricted local minima or maxima of the magnetic energy.

Conclusions. Hall equilibria can be described by a simple variational principle. Long-lived, toroidal fields are not expected in neutron star crusts or other regions where Hall drift is the dominant evolution mechanism. However, other stable configurations do exist, such as current-free poloidal fields and possibly others.
\end{abstract}

Key words. dense matter - magnetic fields - magnetohydrodynamics (MHD) - stars: magnetic fields - stars: neutron - stars: pulsars: general

\section{Introduction}

The Hall drift, namely the advection of magnetic flux by the current associated with it, is important in systems where the magnetic field is strong enough to make the cyclotron frequency comparable or greater than the collision rate, and bulk flow velocities are not much larger than the relative velocity of different charge carriers, associated to the electric current. For some time, it has been realized that this effect might play an important role in the evolution of neutron star magnetic fields, which under some circumstances are large enough for the above conditions to be satisfied, particularly in the solid crust of these stars, in which the electrons are the only mobile charges (Jones 1988; Goldreich \& Reisenegger 1992; Cumming et al. 2004).

The evolution of the magnetic field under the Hall effect is a nonlinear process that has so far eluded a full theoretical understanding. Since the Hall effect conserves the magnetic energy (Urpin \& Shalybkov 1991; Goldreich \& Reisenegger 1992), it has been argued that it will act mainly through the generation of steep magnetic field gradients, on which resistive

^ Current address: Kapteyn Astronomical Institute, University of Groningen, PO Box 800, 9700 AV, Groningen, The Netherlands.

$\star \star$ Permanent address of A. Reisenegger. dissipation can act much more quickly than it would on a smooth field. Goldreich \& Reisenegger (1992) gave general arguments showing that this could happen through a turbulent cascade transferring energy from larger to smaller scales, which was later supported by simulations (Biskamp et al. 1999). On the other hand, Vainshtein et al. (2000) considered an analytic model problem of a plane-parallel slab of matter with a vertical electron density gradient, showing that the evolution of a purely horizontal, everywhere parallel magnetic field can be described by Burgers' equation, producing discontinuities (current sheets) that are smoothed if dissipation is included, but indeed lead to rapid dissipation of the magnetic field.

Other attempts at a better understanding have been numerical. Some authors (Naito \& Kojima 1994; Hollerbach \& Rüdiger 2002, 2004) have computed the evolution of the first few eigenmodes of the magnetic diffusion equation in spherical symmetry, nonlinearly coupled to each other by the Hall effect. Urpin \& Shalybkov (1991) have modelled the evolution of the magnetic field by solving the evolution equation on a grid for a purely toroidal field on a uniform density sphere, again finding that the magnetic field develops sharper gradients, which later dissipate by resistive effects. Simulating the evolution of a poloidal field in a similar way (Shalybkov \& Urpin 1996), energy was found to be 
transferred to a toroidal component and back, complicating the results substantially. Pons \& Geppert (2007) have recently simulated the evolution of both poloidal and toroidal components in a neutron star crust with a code that combines finite differences in the radial direction with a spherical harmonic decomposition in angle, using a realistic description of the radial profile of electron density and electric resistivity and the evolution of the latter. They confirm the transfer of energy back and forth between the poloidal and the toroidal component (especially from the former to the latter), and find regimes along the neutron star evolution when Hall drift or Ohmic diffusion dominate.

In a different approach, Rheinhardt \& Geppert (2002) (see also Geppert \& Rheinhardt 2002; Geppert et al. 2003; Rheinhardt et al. 2004) did a numerical stability analysis on plane-parallel field configurations that do not by themselves evolve under the Hall effect, finding both stable modes (a particular case of which are the well-known "whistler waves") and unstable ones. Based on several examples, they conjecture that, at least in simple geometries, the determining factor for the existence of unstable modes is a sufficiently large second spatial derivative of the unperturbed magnetic field strength.

The present paper reports an analytical study of the evolution of a magnetic field with a possibly realistic, axially symmetric configuration in a solid star with non-uniform electron density. We first give a short re-derivation of the relevant form of the induction equation (Sect. 2) and specialize it to the two independent scalar functions determining the magnetic field in axial symmetry, discussing some of its implications (Sect. 3). Then, we consider the very special case of a purely toroidal magnetic field (Sect. 4). It is shown that, if only the Hall drift is active, the magnetic field evolves independently on each of a family of nested toroidal surfaces, again being determined (for a suitable change of variables) by Burgers' equation (Sect. 4.1). As found by Vainshtein et al. (2000), this leads to the formation of current sheets that can rapidly dissipate, so the field evolves on a Hall time scale to a simple configuration on each of these surfaces, minimizing the magnetic energy while conserving the flux within each toroid (Sect. 4.2). Next (Sect. 5), we consider various aspects of small perturbations and Hall instabilities: we show that a stationary toroidal magnetic field is generally unstable to poloidal perturbations (Sect. 5.1 - up to here, our results were already summarized in Reisenegger et al. 2005) and interpret this through the plane-parallel model considered by previous authors (Sect. 5.2), we discuss the issue of energy conservation in Hall instabilities, show that current-free configurations are stable (Sect. 5.3), and that a current-free, poloidal field supports whistler-like waves (Sect. 5.4). Then, we show that Hall equilibria can be derived from a variational principle, by requiring the magnetic energy to be stationary with respect to small displacements that do not alter the electron density, and use this to argue that some configurations, corresponding to maxima or minima of the magnetic energy subject to these constraints, must be stable with respect to small perturbations evolving solely through the Hall drift (Sect. 6). Finally, we list our main conclusions (Sect. 7).

\section{Induction equation}

In order to clarify our assumptions and notation, we start by re-deriving the equation of motion for the magnetic field in an arbitrary geometry. More general derivations have been given, among others, in Goldreich \& Reisenegger (1992) and Reisenegger et al. (2005).
We consider a material in which the electrons are the only moving particles, embedded in a perfectly rigid, neutralizing background (i.e., an idealized crystal lattice) against which they can occasionally scatter. The steady-state, local average velocity of the electrons is determined by the balance of the Lorentz force against the time-averaged momentum loss through collisions, which yields a generalized Ohm's law,

$\boldsymbol{j}=-n e \boldsymbol{v}=\sigma\left(\boldsymbol{E}+\frac{\boldsymbol{v} \times \boldsymbol{B}}{c}\right)$,

where $\boldsymbol{j}$ is the electric current density, $n,-e$, and $v$ are the number density, charge, and average velocity of the electrons, $\sigma$ is the (scalar) electrical conductivity, $\boldsymbol{E}$ and $\boldsymbol{B}$ are the electric and magnetic field, and $c$ is the speed of light. The evolution of the magnetic field with time $t$ is described by the induction equation, $\partial \boldsymbol{B} / \partial t=-c \nabla \times \boldsymbol{E}$, which, replacing $\boldsymbol{E}$ from Eq. (1), takes the form

$\frac{\partial \boldsymbol{B}}{\partial t}=\nabla \times\left(\boldsymbol{v} \times \boldsymbol{B}-\frac{c \boldsymbol{j}}{\sigma}\right)$.

In the infinite-conductivity limit, this equation asserts that the magnetic field lines can be thought of as drifting along with the electron flow ("Hall drift"). The term involving the conductivity corresponds to deviations from this idealization due to resistive diffusion of the magnetic field.

In the slow-motion limit (i.e., ignoring electromagnetic radiation), the current, and thus the electron velocity, are also related to the magnetic field by Ampère's law, $\boldsymbol{j}=-n e \boldsymbol{v}=c \nabla \times \boldsymbol{B} /(4 \pi)$. This yields a magnetic field evolution law with $\boldsymbol{B}$ as the only dynamical variable,

$\frac{\partial \boldsymbol{B}}{\partial t}=-\nabla \times\left[\frac{c}{4 \pi n e}(\nabla \times \boldsymbol{B}) \times \boldsymbol{B}+\eta \nabla \times \boldsymbol{B}\right]$,

where we have introduced the magnetic diffusivity, $\eta \equiv$ $c^{2} /(4 \pi \sigma)$. The Hall term is quadratic in $\boldsymbol{B}$, which has so far impeded a full theoretical understanding.

\section{Axial symmetry}

We now specialize to the case of an axisymmetric star, described by the standard cylindrical coordinates $R, \phi$, and $z$, with electron density $n(R, z)$. For simplicity, we set $\eta=0$ in this derivation, although below we consider the effects of resistive diffusion. The most general, axisymmetric magnetic field can be decomposed into a toroidal component

$\boldsymbol{B}_{\mathrm{T}}=\mathcal{B}(R, z) \nabla \phi$

and a poloidal component

$\boldsymbol{B}_{\mathrm{P}}=\nabla \mathcal{A}(R, z) \times \nabla \phi$.

This decomposition makes it explicit that the field depends only on two scalar functions, $\mathcal{B}$ and $\mathcal{A}$, and explicitly satisfies the condition of zero divergence independently for both components. We choose to write it in terms of the gradient $\nabla \phi=\hat{\phi} / R$ instead of the unit vector $\hat{\phi}$ in order to make easy use of the identity $\nabla \times \nabla \phi=0$. For future reference, we also write the toroidal and poloidal components of the electron velocity,

$\boldsymbol{v}_{\mathrm{T}}=\frac{c}{4 \pi e n(R, z)} \Delta^{*} \mathcal{A} \nabla \phi$,
$\boldsymbol{v}_{\mathrm{P}}=-\frac{c}{4 \pi e n(R, z)} \nabla \mathcal{B} \times \nabla \phi$, 
where $\Delta^{*} \equiv \nabla^{2}-(2 / R) \partial / \partial R$ is the Grad-Shafranov operator (e.g., Kulsrud 2005, Sect. 4.9).

This shows that the magnetic field lines lie on the surfaces $\mathcal{A}=$ constant, while the current or electron flow lines lie on surfaces $\mathcal{B}=$ constant. If both $\mathcal{A}$ and $\mathcal{B}$ are taken to be zero on the symmetry axis, then $2 \pi \mathcal{A}$ is the poloidal flux enclosed by a given surface $\mathcal{A}=$ constant, whereas $c \mathcal{B} / 2$ is the total current enclosed by the corresponding surface (see also Kulsrud 2005, Sect. 4.9).

Replacing the previous expressions into the induction equation, Eq. (3), and separating toroidal and poloidal components, we obtain the evolution of the two scalar functions,

$$
\begin{aligned}
\frac{\partial \mathcal{B}}{\partial t} & =R^{2}\left[\nabla\left(\chi \Delta^{*} \mathcal{A}\right) \times \nabla \mathcal{A}+\nabla \chi \times \mathcal{B} \nabla \mathcal{B}\right] \cdot \nabla \phi, \\
\frac{\partial \mathcal{A}}{\partial t} & =R^{2} \chi \nabla \mathcal{A} \times \nabla \mathcal{B} \cdot \nabla \phi,
\end{aligned}
$$

where we have introduced the scalar function $\chi(R, z) \equiv$ $c /\left[4 \pi e n(R, z) R^{2}\right]$. These equations show that the toroidal and poloidal components of the field couple strongly to each other in their evolution, so the latter might become quite intricate in the general case. However, there are a few simple but interesting things to be noted.

First, $\mathcal{A}$ does not evolve if the surfaces $\mathcal{A}=$ constant and $\mathcal{B}=$ constant coincide, i.e., one of these scalar functions can be written as a function of the other. In this case, the poloidal components of the magnetic field and the current density are parallel to each other at every point, $\boldsymbol{j}_{\mathrm{P}}=(c / 4 \pi)(\mathrm{d} \mathcal{B} / \mathrm{d} \mathcal{A}) \boldsymbol{B}_{\mathrm{P}}$. They lie in these surfaces, and the coefficient is also constant on each surface. Since there cannot be substantial currents flowing into and out of the star, the poloidal currents on any field line extending out of the star must vanish identically.

This condition, $\boldsymbol{j}_{\mathrm{P}} \times \boldsymbol{B}_{\mathrm{P}}=0$, is equivalent to imposing that the azimuthal component of the Lorentz force is zero. Since neutron stars are born in a fluid state, it might be natural to require that they start in an MHD equilibrium, in which magnetic forces are balanced by fluid forces. In an axially symmetric configuration, the fluid forces cannot have an azimuthal component, therefore naturally leading to this condition.

Second, the previous condition does not ensure that $\mathcal{B}$ will not evolve, so it may not continue to be satisfied at later times as the magnetic field Hall-drifts through the solid (see Reisenegger \& Thompson 2007, for a related discussion for Hall drift in a fluid star). In order to insure that $\mathcal{B}=$ constant as well, one also needs to impose that $\chi\left(\Delta^{*} \mathcal{A}+\mathcal{B} \mathrm{d} \mathcal{B} / \mathrm{d} \mathcal{A}\right)$ is constant on the same surfaces as $\mathcal{A}$ and $\mathcal{B}$. A particular case of a stationary solution is the poloidal field configuration found by Cumming et al. (2004), in which the electron velocity corresponds to a pure rigid-body rotation, so one has $\mathcal{B}=0$ and an angular velocity $\Omega=\chi \Delta^{*} \mathcal{A}=$ constant everywhere.

Aside from this very special case, a purely poloidal initial field $(\mathcal{A} \neq 0, \mathcal{B}=0$ ) will generate a toroidal component as well, because its electron velocity corresponds to a differential rotation that stretches the field lines in the azimuthal direction. However, the converse is not true: if the initial field is purely toroidal, i.e., initially $\mathcal{A}$ is exactly zero, it will remain that way, and only $\mathcal{B}$ will evolve (Urpin \& Shalybkov 1991). The evolution of such a field is much simpler than the general case, but still non-trivial, so we devote the next section to its study.

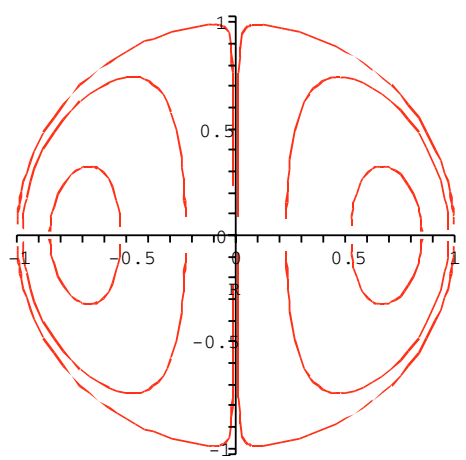

Fig. 1. A meridional cut of a spherical star with an assumed electron density profile $n(r)=n_{0}\left[1-\left(r / r_{0}\right)^{2}\right]$, where $r$ is the (spherical) radial coordinate, $r_{0}$ is the stellar radius, and $n_{0}$ is the electron density at $r=0$. Shown are " $\chi$-surfaces" corresponding to $\chi / \chi_{0}=5,20$, and $10^{4}$, where $\chi_{0}=c /\left(4 \pi e n_{0} r_{0}^{2}\right)$.

\section{Toroidal field}

\subsection{Evolution: burgers equation and current sheets}

The evolution of a purely toroidal magnetic field, now again including a resistive term with axisymmetric diffusivity, $\eta(R, z)$, is given by

$\frac{\partial \mathcal{B}}{\partial t}+w \cdot \nabla \mathcal{B}=R^{2} \nabla \cdot\left(\frac{\eta}{R^{2}} \nabla \mathcal{B}\right)$

with $^{1} w \equiv R^{2} \mathcal{B} \nabla \chi \times \nabla \phi$. Thus, in the absence of dissipation $(\eta=0)$, the quantity $\mathcal{B}$ can be viewed as being advected by the "velocity field" $w$ like a scalar conserved quantity by a hydrodynamic flow. This advection is very different from that of the magnetic flux $\boldsymbol{B}$ by the electron velocity field $\boldsymbol{v}=R^{2} \chi \nabla \phi \times \nabla \mathcal{B}$, which can change the magnitude of the field by compressing or diluting the flux. The velocity field $\boldsymbol{w}$ is clearly perpendicular to $\boldsymbol{B}$ (i.e., poloidal and tangent to surfaces of $\chi(R, z)=$ constant).

Figure 1 shows the surfaces of constant $\chi$ for a particular neutron star model. The singular surface $\chi=+\infty$ is the union of the star's symmetry axis (on which $R=0$ ) and its surface (where $n=0$ ). For all $\chi$ larger than its minimum value $\chi_{\min }$, the " $\chi$-surfaces" are nested surfaces of toroidal topology, while $\chi_{\text {min }}$ defines an equatorial circle.

In the absence of dissipation, the evolution of the magnetic field at any given point depends only on its value at other points on the same $\chi$-surface, not on the values on adjacent surfaces. We define a new coordinate $s$ on each $\chi$-surface by the condition $\partial / \partial s \equiv R^{2} \nabla \chi \times \nabla \phi \cdot \nabla$, so a variation $\mathrm{d} s$ at constant $\chi$ and $\phi$ corresponds to a physical displacement $|\mathrm{d} \boldsymbol{r}|=R|\nabla \chi| \mathrm{d} s$. This allows the field evolution to be written as the dissipationless Burgers equation (Burgers 1940, 1948),

$\mathcal{B}_{t}+\mathcal{B B}_{s}=0$

with the subscripts denoting partial derivatives. The domain of this equation is a closed loop of constant $\chi$ and $\phi$, therefore its boundary conditions must be periodic.

It is well known (Burgers 1940, 1948) that, for a given initial condition $\mathcal{B}(s, 0)=f(s)$, where $f$ is an arbitrary function, Burgers' equation has an implicit, analytic solution,

$\mathcal{B}=f(s-\mathcal{B} t)$

1 There is an ambiguity in the choice of the vector field $w$, in the sense that adding to it another vector field that is everywhere perpendicular to $\nabla \mathcal{B}$ does not change the evolution of $\mathcal{B}$. Our choice is convenient in the sense that the flow lines of $w$ are independent of $\mathcal{B}$ and therefore do not change with time. 
so the value $\mathcal{B}$ of the function at any point $s$ is carried along the surface with "velocity" $\mathrm{d} s / \mathrm{d} t=\mathcal{B}$. The larger values travel faster, so discontinuities are formed when they are about to "overtake" the more slowly moving, smaller values.

The model considered here encompasses two previously studied models as limiting cases. One is a plane-parallel slab with a horizontal magnetic field and vertically decreasing electron density, in which Vainshtein et al. (2000) showed that Burgers' equation governs the field evolution on horizontal planes. The other is a sphere or other axisymmetric body with a toroidal field and uniform electron density, in which simulations (Urpin \& Shalybkov 1991) yield that the magnetic field (initially taken to be symmetric with respect to the equatorial plane) progressively concentrates in one hemisphere, where it develops a strong gradient and eventually gets dissipated. This is easy to understand in terms of our results, as in this case the $\chi$-surfaces are coaxial, cylindrical surfaces, along which the field drifts towards the surface, developing a discontinuity characteristic of Burgers' equation (Araya 2002; Pons \& Geppert 2007).

One feature of our, more general solution is not present in either of these special cases. Since the quantity $\mathcal{B}$ is conserved as it is carried along the $\chi$-surfaces, the magnetic field strength $|\boldsymbol{B}|=|\mathcal{B} \nabla \phi|=|\mathcal{B}| / R$ changes in inverse proportion to the distance to the axis, $R$. In particular, when the flux near the surface of the star is carried from low latitudes to the vicinity of a pole, the field strength increases, as found in simulations (Hollerbach \& Rüdiger 2002, 2004).

In both limiting cases mentioned above, one is forced to adopt somewhat arbitrary boundary conditions, in the first case because the slab is infinite, in the other because the electron density drops abruptly to zero at the surface of the star, where the $\chi$-surfaces end. This is not a problem in more realistic cases, in which the electron density decays continuously to zero at the stellar surface, and $\chi$-surfaces are closed and therefore have no boundaries. Of course, a real neutron star is not fully solid, but has a fluid core, which will be threaded by the $\chi$-surfaces. Thus, magnetic flux will generally be transported back and forth between the fluid core and the solid crust, an effect that will require understanding the Hall drift in fluid matter, which is addressed by Reisenegger \& Thompson (2007).

In order to find the time of occurrence of the discontinuities in the magnetic field, one may consider the partial derivative of Eq. (12) with respect to $s, \mathcal{B}_{s}=\left(1-\mathcal{B}_{s} t\right) f^{\prime}(s-\mathcal{B} t)$, from which one obtains $\mathcal{B}_{s}=f^{\prime} /\left(1+t f^{\prime}\right)$. A discontinuity $\left(\mathcal{B}_{s} \rightarrow \pm \infty\right)$ is formed at time $t_{\text {disc }}=1 / \max \left(-f^{\prime}\right)$, at the comoving point where the initial spatial derivative had the largest, negative slope. (Note that the Hall effect is not invariant under spatial reflection.)

These discontinuities in the magnetic field strength correspond to sufaces of infinite current density. In practice, before a "current sheet" becomes singular, resistive dissipation must occur, even for arbitrarily small $\eta$. This dissipation occurs in a thin layer, whose width is $\approx \eta /(R|\nabla \chi| \Delta \mathcal{B})$, where $\Delta \mathcal{B}$ is the difference in the values of $\mathcal{B}$ across the (near) discontinuity (Vainshtein et al. 2000). However, the rate of dissipation depends exclusively on the rate at which the Hall effect transports the scalar variable $\mathcal{B}$ (related to the magnetic flux) to the current sheet.

\subsection{Conserved quantities}

From the induction equation for a toroidal field (Eq. (10)), and using the identity

$\nabla \cdot\left(\frac{w}{R^{2} \mathcal{B}}\right)=\nabla \cdot(\nabla \chi \times \nabla \phi)=0$, we can show that any scalar function $F(\mathcal{B})$ defines a "density",

$\rho \equiv \frac{F^{\prime}}{R^{2}}$

and a "flux",

$J \equiv \frac{\mathcal{B}}{R^{2}} \frac{\mathrm{d}}{\mathrm{d} \mathcal{B}}\left(\frac{F}{\mathcal{B}}\right) w-\frac{\eta}{R^{2}} F^{\prime \prime} \nabla \mathcal{B}$

that satisfy a continuity equation

$\frac{\partial \rho}{\partial t}+\nabla \cdot \boldsymbol{J}=-\frac{\eta}{R^{2}} F^{\prime \prime \prime}(\nabla \mathcal{B})^{2}$

where primes $\left({ }^{\prime}\right)$ denote derivatives of $F$ with respect to its argument, $\mathcal{B}$. Thus, the physical quantity whose density is $\rho$ is transported by the Hall drift in the direction of $w$, i.e., along $\chi$-surfaces, and by Ohmic diffusion in the direction of decreasing $\mathcal{B}$, while it is being destroyed by the right-hand-side term, which is also related to Ohmic diffusion. (For definiteness, in this discussion we assumed that $\mathcal{B}, F^{\prime}, F^{\prime \prime}$, and $F^{\prime \prime \prime}$ are all $>0$.) Thus, in the absence of Ohmic diffusion $(\eta=0)$, the volume integral over the toroid bounded by a $\chi$-surface,

$Q\left(\chi_{0} ; t\right) \equiv \int_{\chi(r)<\chi_{0}} \rho(\mathcal{B}[\boldsymbol{r}, t]) \mathrm{d} V$,

is constant in time. We consider two specific examples of such conservation laws, namely those of magnetic flux and of magnetic energy.

In order to obtain magnetic flux conservation, we must set $F=\mathcal{B}^{2} / 2$. For this choice, the right-hand-side of Eq. (16) vanishes, indicating that magnetic flux can be transported by both Hall drift and Ohmic diffusion, but neither created nor destroyed, and the continuity equation can be rewritten in terms of the magnetic flux density $B=\mathcal{B} / R$ as

$$
\begin{gathered}
\frac{\partial B}{\partial t}+\frac{\partial}{\partial R}\left[\frac{1}{2} B w_{R}-\frac{\eta}{R} \frac{\partial}{\partial R}(R B)\right] \\
+\frac{\partial}{\partial z}\left[\frac{1}{2} B w_{z}-\eta \frac{\partial B}{\partial z}\right]=0,
\end{gathered}
$$

which can be interpreted as a two-dimensional continuity equation for $B$ on the Cartesian $R-z$ plane.

For magnetic energy conservation, we set $F=\mathcal{B}^{3} /(24 \pi)$, obtaining

$$
\begin{aligned}
\frac{\partial}{\partial t}\left[\frac{1}{8 \pi}\left(\frac{\mathcal{B}}{R}\right)^{2}\right] & +\nabla \cdot\left[\left(\frac{\mathcal{B}}{R}\right)^{2} \frac{w}{12 \pi}-\frac{\eta \mathcal{B}}{4 \pi R^{2}} \nabla \mathcal{B}\right] \\
& =-\frac{\eta}{4 \pi R^{2}}(\nabla \mathcal{B})^{2} .
\end{aligned}
$$

The right-hand-side is negative-definite, so magnetic energy can be destroyed by Ohmic diffusion, but not created. We note that the magnetic energy flux vector obtained here is generally different (in its Hall-drift part) from the standard Poynting vector,

$\boldsymbol{S}=\frac{c}{4 \pi} \boldsymbol{E} \times \boldsymbol{B}=-\frac{\chi \mathcal{B}}{4 \pi} \nabla \mathcal{B} \times \nabla \phi-\frac{\eta \mathcal{B}}{4 \pi R^{2}} \nabla \mathcal{B}$.

However, their divergence, which determines their physical effects, is the same.

In the scenario discussed in the previous section, in which Hall drift dominates and Ohmic dissipation is only significant in thin current sheets, we expect no large-scale transport of magnetic flux or energy across $\chi$-surfaces, but only a redistribution 
on each surface by Hall drift and local Ohmic dissipation in the current sheets, which causes the magnetic energy to decrease while keeping the magnetic flux constant. Thus, the asymptotically resulting field configurations $\boldsymbol{B}_{0}=\mathcal{B}(\chi) \nabla \phi$ are minima of the magnetic energy subject to conservation of flux in the region within each $\chi$-surface, and are the only toroidal equilibrium states as far as the Hall effect is concerned. We note that, like the stationary, poloidal field found by Cumming et al. (2004), corresponding to a rigidly rotating electron fluid, these configurations are also not force-free, in the sense that $\boldsymbol{j} \times \boldsymbol{B} \neq 0$. In the long term, of course, diffusion will allow magnetic flux transport across $\chi$-surfaces, eventually leading to magnetic field decay.

\section{Perturbations and instability}

\subsection{Poloidal perturbations of the toroidal equilibrium field}

We now study the stability of this stationary, toroidal magnetic field to a small, poloidal perturbation, $\boldsymbol{B}_{1}$. The associated velocity field $\boldsymbol{v}_{1}$ is toroidal, therefore $\boldsymbol{v}_{1} \times \boldsymbol{B}_{0}=0$, and the linearized evolution equation for the perturbation reduces to

$\frac{\partial \boldsymbol{B}_{1}}{\partial t}=\nabla \times\left(\boldsymbol{v}_{0} \times \boldsymbol{B}_{1}\right)$

which implies that the field lines of the perturbation are carried along by the background electron flow field, $\boldsymbol{v}_{0}=-(c / 4 \pi n e) \nabla \times$ $\boldsymbol{B}_{0}$, and the perturbation field $\boldsymbol{B}_{1}$ remains poloidal.

Thus, it can be written as $\boldsymbol{B}_{1}=\nabla \mathcal{A}(\chi, s, t) \times \nabla \phi$, with $|\nabla \mathcal{A}| \ll|\mathcal{B}|$. Replacing into the above equation, one finds that the potential evolves according to

$\frac{\partial \mathcal{A}}{\partial t}=-\boldsymbol{v}_{0} \cdot \nabla \mathcal{A}=\chi \frac{\mathrm{d} \mathcal{B}}{\mathrm{d} \chi} \frac{\partial \mathcal{A}}{\partial s}$,

i.e., the scalar quantity $\mathcal{A}$ is also carried along $\chi$-surfaces by the unperturbed electron flow. Thus, it can generally be written in terms of its initial condition as

$\mathcal{A}(\chi, s, t)=\mathcal{A}(\chi, s+\chi[\mathrm{d} \mathcal{B} / \mathrm{d} \chi] t, 0)$.

Initially, the smallness of $\boldsymbol{B}_{1}$ forces $\mathcal{A}$ to be a smooth function of position, taking similar values on adjacent points of different $\chi$-surfaces. However, when the circulation periods of the electrons around different $\chi$-surfaces are different, each point comoving with them will come close to others that were initially far away, so the values of $\mathcal{A}$ within a vicinity of fixed size around a given point will become progressively different, leading to a linearly increasing perturbation field. Another way of viewing this process is by realizing that the magnetic field lines of the perturbation are stretched as different parts are carried by electron currents circulating with different periods on different $\chi$-surfaces. A simulation of this evolution is shown in Fig. 2.

As the function $\mathcal{A}(\chi, s, t)$ is periodic, the perturbed field oscillates in time, periodically for the component perpendicular to the $\chi$-surfaces, and with increasing amplitude for the tangential component. A similar, oscillating behavior has been observed in numerical simulations (Hollerbach \& Rüdiger 2002, 2004; Shalybkov \& Urpin 1996). However, given their more complex geometries, a direct connection is difficult to establish.

\subsection{Plane-parallel analog}

In order to understand the relation of the instability found analytically by us to those found numerically by Rheinhardt \& Geppert (2002) in a plane-parallel slab and later discussed by
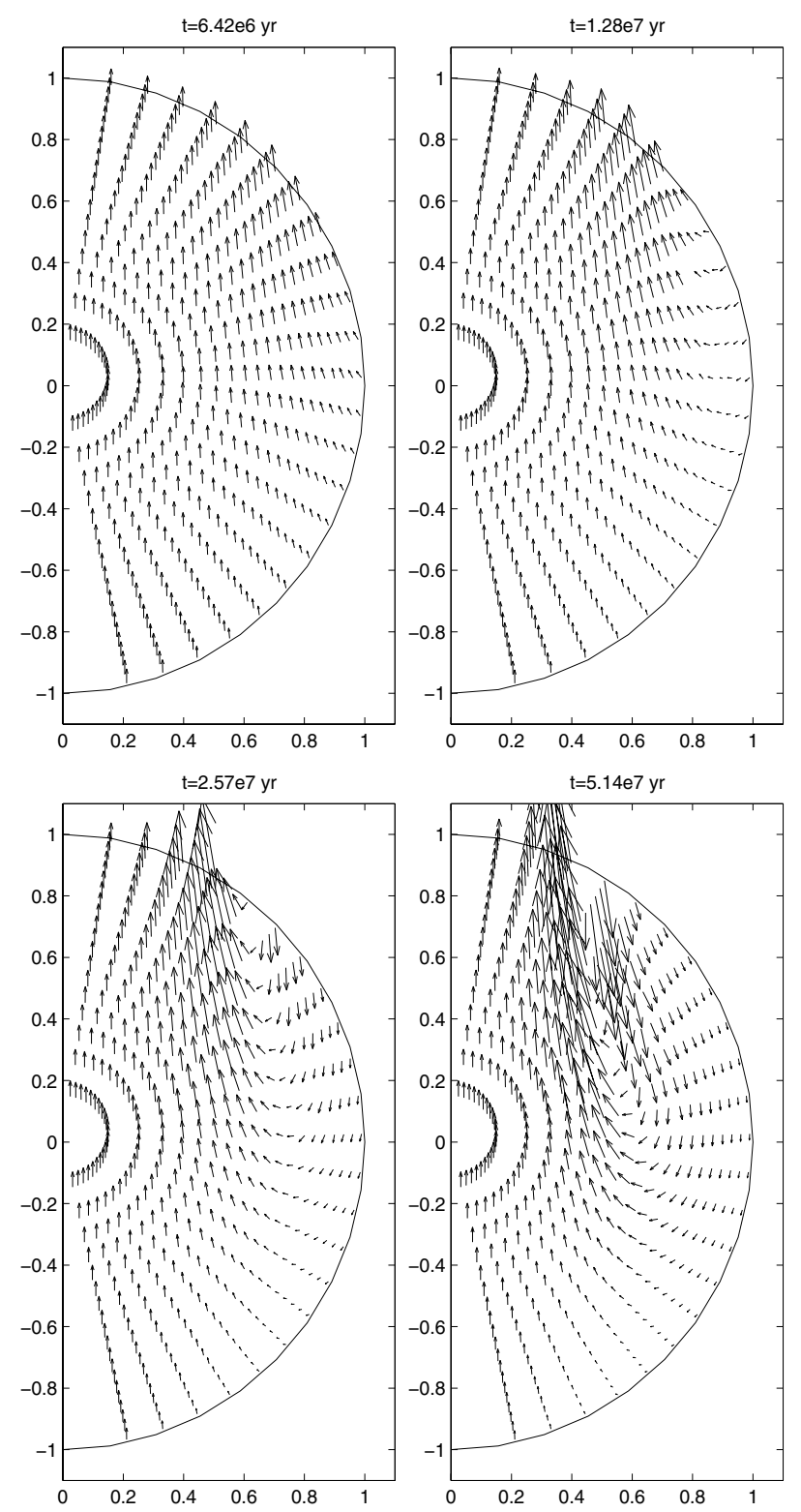

Fig. 2. The evolution of a weak poloidal magnetic field component affected by a much stronger toroidal background field. Shown are the poloidal field vectors on a cut through a star with electron density profile $n(r)=n_{0}\left[1-\left(r / r_{0}\right)^{2}\right]$, with central density $n_{0}=10^{36} \mathrm{~cm}^{-3}$, and stellar radius $r_{0}=10 \mathrm{~km}$. The background toroidal field has the form $\boldsymbol{B}_{0}=\mathcal{B}(\chi) \nabla \phi$, where we chose $\mathcal{B}(\chi)=b_{0} r_{0}\left(\chi_{0} / \chi\right)^{2}$, with $b_{0}=10^{14} \mathrm{G}$, $\chi=c /\left(4 \pi n e R^{2}\right)$, and $\chi_{0}$ its minimum value. The initial poloidal field was chosen uniform and pointing along the symmetry axis. The evolutionary times scale $\propto n_{0} r_{0}^{2} / B_{0}$.

Cumming et al. (2004), we consider the plane-parallel geometry studied by these authors, in which a horizontal field varying nonlinearly with depth generates different velocities at different depths, stretching a small, vertical field component.

We take the vertical coordinate to be $z$, and consider a background magnetic field $\boldsymbol{B}_{0}=f(z) \hat{x}$, which generates an electron velocity $\boldsymbol{v}_{0}=-c f^{\prime}(z) /[4 \pi n(z) e] \hat{y}$, where primes denote derivatives with respect to $z$. If an additional, uniform, vertical field $B_{1 z}$ is introduced, it causes the linear growth of a component in the $y$ direction, $\partial B_{1 y} / \partial t=-\left[c B_{1 z} f^{\prime}(z) /(4 \pi n(z) e)\right]^{\prime}$. In some sense, this is a particular version or limiting case of the instabilities found by Rheinhardt \& Geppert (2002), with vanishing horizontal wave vector. However, it does not appear in their calculation, 
as this field perturbation cannot be written in terms of two sinusoidally varying potentials, as done in their Eqs. (4) and (5). For that representation, a magnetic field perturbation with vanishing horizontal wave vector would be identically zero. Nevertheless, in our solution as in that work, if the electron density is taken to be constant, the growth of the perturbation depends directly on the second spatial derivative of the background field strength, $f^{\prime \prime}(z)$, suggesting that we are in fact seeing the same kind of instability. In our model, we confirm the conclusion of Cumming et al. (2004) that this finite second derivative is caused by a sheared electron flow velocity, which bends the vertical field component, creating an additional horizontal field.

Curiously, in this simple geometry, we can actually calculate the nonlinear development of the instability. We consider a field with three components of arbitrary strength: $B_{x}(z, t)$, which is initially the "background" field, but is now allowed to evolve arbitrarily large perturbations; the initially small (or vanishing), but growing component $B_{y}(z, t)$; and the vertical component $B_{z}$, which represents the initial perturbation. For this choice, the components of the induction equation become

$$
\begin{aligned}
\frac{\partial B_{x}}{\partial t} & =\frac{\partial}{\partial z}\left(\frac{c B_{z}}{4 \pi n(z) e} \frac{\partial B_{y}}{\partial z}\right), \\
\frac{\partial B_{y}}{\partial t} & =-\frac{\partial}{\partial z}\left(\frac{c B_{z}}{4 \pi n(z) e} \frac{\partial B_{x}}{\partial z}\right), \\
\frac{\partial B_{z}}{\partial t} & =0 .
\end{aligned}
$$

These are the equations for "helicons" or "whistler waves" of arbitrary amplitude propagating along the $z$ axis. For $n(z)=$ constant, they have a dispersion relation $\omega=\left[c B_{z} /(4 \pi n e)\right] k^{2}$, where $\omega$ is the frequency and $k$ is the magnitude of the wave vector (which points in the $\pm z$ direction). One particular solution is

$$
\begin{aligned}
& B_{x}(z, t)=B_{m} \cos (k z) \cos (\omega t), \\
& B_{y}(z, t)=B_{m} \cos (k z) \sin (\omega t), \\
& B_{z}=\text { constant }
\end{aligned}
$$

where the horizontal component initially points along the $x$ axis, then turns to the $y$ axis at a rate determined by $B_{z}$, and eventually turns around completely. Thus, the evolution of the field in this case is strictly periodic (with the period equal to the Hall time scale of the "small" component $B_{z}$ ) and does not lead to dissipation on a Hall time scale. If Ohmic diffusion is introduced, the amplitude of the horizontal component will decay exponentially, on the resistive time scale $1 /\left(\eta k^{2}\right)$. However, we suspect that this "clean" behavior is a peculiarity of this very symmetric configuration, and will not hold in more realistic cases, including the simulations of Rheinhardt \& Geppert (2002).

\subsection{Energy conservation in Hall instabilities}

In this section, we would like to clarify an issue that was raised, but in our view not fully clarified, by both Rheinhardt \& Geppert (2002) and Cumming et al. (2004), namely the conservation of energy in the growth of Hall instabilities.

In any slow evolution of a magnetic field, one can use the induction equation and Ampére's law to show that the magnetic energy density $u=B^{2} /(8 \pi)$ satisfies

$$
\frac{\partial u}{\partial t}+\nabla \cdot S=-j \cdot \boldsymbol{E}
$$

with the Poynting flux vector $\boldsymbol{S}=(c / 4 \pi) \boldsymbol{E} \times \boldsymbol{B}$. For pure Hall drift, the electric field, $\boldsymbol{E}=\boldsymbol{j} \times \boldsymbol{B} /(n e)$, is perpendicular to the current, so the right-hand side vanishes and the magnetic energy satisfies an exact conservation law (Goldreich \& Reisenegger 1992).

If we decompose the magnetic field, $\boldsymbol{B}=\boldsymbol{B}_{0}+\boldsymbol{B}_{1}$, where $\boldsymbol{B}_{0}$ is the constant or slowly varying "background field", whereas $\boldsymbol{B}_{1}$ is a small, more rapidly varying "perturbation", each can be taken to satisfy an (exact) induction equation, $\partial \boldsymbol{B}_{\alpha} / \partial t=-c \nabla \times$ $\boldsymbol{E}_{\alpha}$, where

$$
\begin{aligned}
& \boldsymbol{E}_{0}=\frac{1}{n e c} \boldsymbol{j}_{0} \times \boldsymbol{B}_{0}, \\
& \boldsymbol{E}_{1}=\frac{1}{n e c}\left(\boldsymbol{j}_{0} \times \boldsymbol{B}_{1}+\boldsymbol{j}_{1} \times \boldsymbol{B}_{0}+\boldsymbol{j}_{1} \times \boldsymbol{B}_{1}\right),
\end{aligned}
$$

and $\boldsymbol{j}_{\alpha}=(c / 4 \pi) \nabla \times \boldsymbol{B}_{\alpha}$, for $\alpha=0,1$. The magnetic energy density can be decomposed into three parts, $u=u_{0}+u_{1}+u_{2}$, where $u_{0}=B_{0}^{2} /(8 \pi)$ and $u_{2}=B_{1}^{2} /(8 \pi)$ are positive-definite, while $u_{1}=\boldsymbol{B}_{0} \cdot \boldsymbol{B}_{1} /(4 \pi)$ is not. For $u_{0}$, the derivation above can be retraced to show that it satisfies an exact conservation law. However, the evolution of $u_{1}$ and $u_{2}$ yields

$$
\begin{aligned}
& \frac{\partial u_{1}}{\partial t}+\nabla \cdot \boldsymbol{S}_{1}=-\frac{1}{n e c} \boldsymbol{j}_{0} \cdot \boldsymbol{j}_{1} \times \boldsymbol{B}_{1}, \\
& \frac{\partial u_{2}}{\partial t}+\nabla \cdot \boldsymbol{S}_{2}=\frac{1}{n e c} \boldsymbol{j}_{0} \cdot \boldsymbol{j}_{1} \times \boldsymbol{B}_{1},
\end{aligned}
$$

where $\boldsymbol{S}_{1}=(c / 4 \pi)\left(\boldsymbol{E}_{1} \times \boldsymbol{B}_{0}+\boldsymbol{E}_{0} \times \boldsymbol{B}_{1}\right)$ and $\boldsymbol{S}_{2}=(c / 4 \pi) \boldsymbol{E}_{1} \times \boldsymbol{B}_{1}$. Thus, the volume integrals of the two pieces, $\int u_{1}$ and $\int u_{2}$, are not individually conserved, but their sum, $\int\left(u_{1}+u_{2}\right)$, is conserved. To linear order in $\boldsymbol{B}_{1}$, only $\int u_{1}$ is non-zero, and it is conserved. To quadratic order, $\int u_{2}$ can increase (in the case of an instability), being balanced by a corresponding decrease in $\int u_{1}$, which becomes progressively more negative. Thus, instabilities can only occur if, for some choices of the perturbation field $\boldsymbol{B}_{1}$, the spatial integral of the right-hand side of Eq. (36) is positive. Clearly, this will not happen for current-free field configurations $\left(j_{0}=0\right)$, for which this right-hand side vanishes identically and no growth will occur. Somewhat more generally, Cumming et al. (2004) have shown that for their stationary configurations, in which the current density is such that all the electrons rotate as a rigid body, the volume-integral of the right-hand side is zero for all perturbations that vanish on the boundaries, therefore also proving their stability.

\subsection{Current-free, poloidal background field}

An application of the result of Sect. 5.3 is given by the following, plausible scenario for the evolution of the magnetic field in a neutron star. The field initially has an approximately axisymmetric configuration with both poloidal and toroidal components that are supported by currents that flow partly in the solid crust and partly in the fluid core of the star. In the core, the conductivity is very high, the Hall drift is ineffective (Reisenegger \& Thompson 2007), and ambipolar diffusion not fast enough, so the field is essentially frozen in some MHD-stable configuration (Braithwaite \& Spruit 2006). In the solid crust, Ohmic dissipation is more effective, particularly if aided by Hall drift, and one might expect the currents to decay, so the field might end up in a current-free configuration, which, by the arguments of the previous section, is stable under the Hall drift.

In order to examine the evolution of the small perturbations, we use our earlier notation, with the background field written 
as $\boldsymbol{B}_{0}=\nabla \mathcal{A}_{0} \times \nabla \phi$, with $\mathcal{B}_{0}=0$ (no toroidal component) and $\Delta^{*} \mathcal{A}_{0}=0$ (no current). It clearly does not evolve through Hall drift, since $\partial \mathcal{A}_{0} / \partial t=0$. Adding a small perturbation, the latter will evolve as

$$
\begin{aligned}
\frac{\partial \mathcal{B}_{1}}{\partial t} & =R^{2} \boldsymbol{B}_{0} \cdot \nabla\left(\chi \Delta^{*} \mathcal{A}_{1}\right)=\frac{1}{\chi} \frac{\partial}{\partial \zeta}\left(\chi \Delta^{*} \mathcal{A}_{1}\right), \\
\frac{\partial \mathcal{A}_{1}}{\partial t} & =-\chi R^{2} \boldsymbol{B}_{0} \cdot \nabla \mathcal{B}_{1}=-\frac{\partial \mathcal{B}_{1}}{\partial \zeta}
\end{aligned}
$$

where we have defined the coordinate $\zeta$ along field lines of $\boldsymbol{B}_{0}$ by the condition $\partial / \partial \zeta \equiv \chi R^{2} \boldsymbol{B}_{0} \cdot \nabla=\left(c \boldsymbol{B}_{0} / 4 \pi n e\right) \cdot \nabla$, analogous to the definition of $s$ on $\chi$-surfaces in Sect. 4 .

One can combine the two equations, eliminating $\mathcal{B}_{1}$ and obtaining

$\frac{\partial^{2} \mathcal{A}_{1}}{\partial t^{2}}=-\frac{\partial}{\partial \zeta}\left[\frac{1}{\chi} \frac{\partial}{\partial \zeta}\left(\chi \Delta^{*} \mathcal{A}_{1}\right)\right]$.

This appears to be an hyperbolic differential equation with wavelike solutions corresponding to helicons or whistler waves travelling along the field lines of $\boldsymbol{B}_{0}$. In the WKB approximation (small-wavelength perturbations), $\boldsymbol{B}_{0}$ can be considered as uniform, $\Delta^{*}$ reduces to the Laplacian, and this equation is exactly the whistler wave equation in a uniform background field. The behavior of long-wavelength perturbations may be more complex, but the arguments given above ensure that they will also be stable.

The more general, "uniformly rotating" configuration of Cumming et al. (2004) has $\boldsymbol{v}_{0}=\boldsymbol{\Omega} \times \boldsymbol{r}$, and its field is therefore still poloidal, but not current-free. Since $\chi \Delta^{*} \mathcal{A}_{0}=\Omega=$ constant (although non-zero), its perturbations satisfy exactly the same Eqs. (37) through (39). This is consistent with the energetic argument for it being stable as well.

This means that the current-free poloidal field (and its generalization, the "uniformly rotating" field of Cumming et al. 2004) is indeed stable under the Hall drift. The field in a neutron-star crust might be able to settle into such a state, which would remain in this form as long as the currents supporting it in the core do not change.

\section{Variational principle}

Since the Hall drift conserves magnetic energy, it is tempting to search for a variational principle that would yield Hall equilibria, and in fact a fairly simple and natural one exists. Consider stationary points of the magnetic energy $U=\int B^{2} /(8 \pi)$ subject to magnetic field perturbations $\delta \boldsymbol{B}=\nabla \times(\boldsymbol{\xi} \times \boldsymbol{B})$ that are due to an infinitesimal displacement field $\boldsymbol{\xi}$ that does not change the electron density, i.e., $\nabla \cdot(n \boldsymbol{\xi})=0$. The latter condition implies that the displacement field can be written as $\boldsymbol{\xi}=(1 / n) \nabla \times \boldsymbol{a}$, where $\boldsymbol{a}$ is an arbitrary vector field. This allows a perturbation of the magnetic energy density to be written as

$$
\begin{aligned}
\delta u= & \frac{\boldsymbol{B} \cdot \delta \boldsymbol{B}}{4 \pi} \\
= & \nabla \cdot\left[\frac{1}{4 \pi}(\boldsymbol{\xi} \times \boldsymbol{B}) \times \boldsymbol{B}+\frac{1}{c n}(\boldsymbol{j} \times \boldsymbol{B}) \times \boldsymbol{a}\right] \\
& -\boldsymbol{a} \cdot \nabla \times\left(\frac{\boldsymbol{j} \times \boldsymbol{B}}{c n}\right) .
\end{aligned}
$$

Integrating over the volume, the divergence term becomes a surface integral that can be made vanish by requiring that the normal components of $\boldsymbol{j}, \boldsymbol{B}$ and $\boldsymbol{\xi}$ all vanish on the surface. (The former two are required in order to ensure that the energy in the volume is conserved under Hall drift, the latter for consistency with the condition of not changing the electron density.) With these conditions, in order to have $\delta U=0$ for an otherwise arbitrary vector field $\boldsymbol{a}$, we must have $\nabla \times(\boldsymbol{j} \times \boldsymbol{B} / n)=0$, which is also the condition to have no Hall drift of the magnetic field. (It can be shown that, for these perturbations and the adopted boundary conditions, the magnetic helicity $\int \boldsymbol{A} \cdot \boldsymbol{B}$ is automatically conserved, i.e., our constraint is stronger than that of requiring helicity conservation.)

Put in a different way, our result states that, if the magnetic field is such that it is not changed by the Hall drift, $\boldsymbol{\xi} \propto(1 / n) \nabla \times$ $\boldsymbol{B}$, then its energy will not change under the more general class of displacements $\boldsymbol{\xi}=(1 / n) \nabla \times \boldsymbol{a}$, with arbitrary $\boldsymbol{a}$.

Presumably, a subset of the configurations at which the magnetic energy is stationary, subject to the constraints discussed, will be maxima or minima of the magnetic energy, i.e., to order $\xi^{2}$ or higher, all perturbations will cause magnetic energy changes of the same sign. For such configurations, there will be only a small set of nearby configurations with a similar energy, and thus they will be stable under Hall drift. The other configurations at which the magnetic energy is stationary will correspond to saddle points, inflection points, etc., whose energy is the same as that of a large set of other configurations that can be obtained by continuously deforming the magnetic field structure. We expect that these will generally be Hall-unstable, in the sense that a small, initial perturbation can eventually make them evolve into a very different configuration.

\section{Conclusions}

We have found some interesting results regarding the Hall-drift evolution of an axially symmetric magnetic field in solid matter, in which electrons are the only mobile particles:

1. We have characterized "Hall equilibrium" configurations that do not evolve under the Hall drift (Sect. 3).

2. We have found that an exactly toroidal field evolves discontinuities described by the Burgers equation, which dissipate on the Hall drift timescale (Sect. 4.1), as in the planeparallel case studied earlier by Vainshtein et al. (2000). The field evolves into a stationary state that minimizes magnetic energy subject to flux conservation within a set of nested, toroidal " $\chi$-surfaces" (Sect. 4.2).

3. However, we found these stationary, toroidal fields to be unstable to poloidal perturbations (Sect. 5.1), making them an unrealistic model for the fields of neutron stars. The instability involved is due to shearing of the perturbation field by the background electron velocity, and thus closely related to those studied by Rheinhardt \& Geppert (2002) and Cumming et al. (2004).

4. We have discussed the issue of energy conservation in Hall instabilities, giving a criterion for their occurrence and showing that current-free fields are stable (Sect. 5.3). We applied it to argue that a current-free, poloidal field in the crust, supported by axially symmetric currents flowing only in the core of the star, might represent a long-lived magnetic configuration of a neutron star (Sect. 5.4).

5. Finally, we showed that a Hall equilibrium is a stationary point for the magnetic energy, subject to displacements that do not alter the electron density, and used this to argue that maxima or minima of the magnetic energy with respect to such perturbations will be stable under the Hall drift (Sect. 6). 
Acknowledgements. This work was supported by FONDECYT Regular Grants 1020840 (A.R. and J.P.P.), 1020844 (R.B. and P.A.A.), 1060644 (A.R.), and 1060651 (R.B.). J.P.P. is also supported by a doctoral fellowship at Pontificia Universidad Católica de Chile (PUC). A visit by D.L. to PUC was made possible by FONDECYT International Cooperation Grant 7020840. The authors thank R. Fernández, U. Geppert, M. Lyutikov, H. Spruit, and C. Thompson for useful discussions.

\section{References}

Araya, P. A. 2002, Informe de Práctica de Licenciatura (undergraduate thesis), Pontificia Universidad Católica de Chile

Biskamp, D., Schwarz, E., Zeiler, A., Celani, A., \& Drake, J. F. 1999, Phys. Plasmas, 6, 751

Braithwaite, J., \& Spruit, H. C. 2004, Nature, 431, 819

Braithwaite, J., \& Nordlund, A. 2006, A\&A, 450, 1077

Braithwaite, J., \& Spruit, H. 2006, A\&A, 450, 1097

Burgers, J. M. 1940, Proc. Roy. Neth. Acad. Sci. (Amsterdam), 43, 2

Burgers, J. M. 1948, Adv. Appl. Mech., 1, 171

Cumming, A., Arras, P., \& Zweibel, E. 2004, ApJ, 609, 999
Geppert, U., \& Rheinhardt, M. 2002, A\&A, 392, 1015

Geppert, U., Rheinhardt, M., \& Gil, J. 2003, A\&A, 412, L33

Goldreich, P., \& Reisenegger, A. 1992, ApJ, 395, 250

Hollerbach, R., \& Rüdiger, G. 2002, MNRAS, 337, 216

Hollerbach, R., \& Rüdiger, G. 2004, MNRAS, 347, 1273

Jones, P. B. 1988, MNRAS, 233, 875

Kulsrud, R. 2005, Plasma Physics for Astrophysics (Princeton: Princeton University Press)

Naito, T., \& Kojima, Y. 1994, MNRAS, 266, 597

Pons, J., \& Geppert, U. 2007, [arXiv: astro-ph/0703267]

Reisenegger, A., Prieto, J. P., Benguria, R., Lai, D., \& Araya, P. A. 2005, in

Magnetic Fields in the Universe: From Laboratory and Stars to Primordial

Structures, ed. E. M. de Gouveia dal Pino, G. Lugones, \& A. Lazarian, AIP

Conf. Proc., 784, 263

Reisenegger, A., \& Thompson, C. 2007, in preparation

Rheinhardt, M., \& Geppert, U. 2002, Phys. Rev. Lett., 88, 101103

Rheinhardt, M., Konenkov, D., \& Geppert, U. 2004, A\&A, 420, L33

Shalybkov, D. A., \& Urpin, V. A. 1996, A\&A, 321, 685

Urpin, V. A., \& Shalybkov, D. A. 1991, Sov. Phys. JETP, 73, 703

Vainshtein, S. I., Chitre, S. M., \& Olinto, A. V. 2000, Phys. Rev. E, 61, 4422 